\title{
Time domain simulation of power systems with different time scales
}

\author{
Valeriu Savcenco $^{1}$, Bertrand Haut ${ }^{2}$, E. Jan W. ter Maten ${ }^{1,3}$, and Robert M.M. Mattheij ${ }^{1}$ \\ 1 Eindhoven University of Technology, P.O. Box 513, 5600 MB Eindhoven, The Netherlands v.savcenco@tue.nl, \\ r.m.m.mattheij@tue.nl,e.j.w.ter.maten@tue.nl \\ 2 Tractebel Engineering S.A, Avenue Ariane 7, 1200 Brussels, Belgium bertrand.haut egdf suez.com \\ 3 NXP Semiconductors B.V., High Tech Campus 46, 5656 AE Eindhoven, The Netherlands \\ jan.ter.maten@nxp.com
}

Summary. The time evolution of power systems is modeled by a system of differential and algebraic equations. The variables involved in the system may exhibit different time scales. In standard numerical time integration methods the most active variables impose the time step for the whole system. We present a strategy, which allows the use of different, local time steps over the variables. The partitioning of the components of the system in different classes of activity is based on the topology of the power system.

\section{Introduction}

Modeling of power systems results in large differential-algebraic systems. These systems are built from the equations describing the network, the generators, the voltage regulators, the speed governors and the dynamic shunt loads. All together they form a non-linear system in semi-explicit form

$$
\begin{gathered}
y^{\prime}=f(t, y, z), \\
0=g(t, y, z),
\end{gathered}
$$

with initial values $y(0)=y_{0}$ and $z(0)=z_{0}$, such that $g\left(t_{0}, y_{0}, z_{0}\right)=0$. It is assumed that the matrix $\frac{\partial g}{\partial z}$ is non singular and therefore system (1) has index one.

Time domain simulation is an important application for the dynamic security assessment of power systems [4]. The components involved in the systems are known to exhibit a wide range of time scales. To solve such problems multirate time stepping methods can be efficient $[2,3,5]$. With such methods different solution components can be integrated with different time steps.

\section{Multirate time stepping}

In this paper it will be assumed that the variables of the system (1) can be partitioned into fast and slow

$$
y=\left[y_{\text {fast }}, y_{\text {slow }}\right] \quad \text { and } \quad z=\left[z_{\text {fast }}, z_{\text {slow }}\right] .
$$

Our multirate time stepping strategy can be described as follows. For a given global time step $\tau=t_{n}-t_{n-1}$, we first compute a tentative approximation at the time level $t_{n}$ for the both fast and slow variables. We accept the computed numerical solution for the slow components, while for the fast components the computation is redone with smaller time steps. During this refinement computation the subsystem

$$
\begin{array}{r}
y_{\text {fast }}^{\prime}=f_{\text {fast }}\left(t, y_{\text {fast }}, z_{\text {fast }}, \omega\right), \\
0=g_{\text {fast }}\left(t, y_{\text {fast }}, z_{\text {fast }}, \omega\right)
\end{array}
$$

is solved, where $\omega$ denotes the already computed values of the slow variables. During the refinement stage, values at the intermediate time levels of the slow components might be needed. These values can be obtained by interpolation.

\section{Partitioning Strategy}

Partitioning of the variables in slow and fast can be fixed and given in advance, or it can vary in time and should be performed automatically during the time integration process.

In this section we present a strategy for automatic partitioning of the differential and algebraic variables. This strategy is based on the local time variation of the numerical solution of the system and on the topology of the power system.

We first perform a single step with step size $\tau$ and using an error estimator we determine the variables which do not satisfy the criterion

$$
e_{i}<\text { Tol }
$$

where $e_{i}$ is the estimated local error for the variable $i$ and $T o l$ is a given tolerance. These variables will be called fast. To allow for accurate computation of the fast variables, during the refinement stage, we also recompute the slow variables which are strongly coupled to the fast ones. The whole set of components for which refinement is performed is denoted by $\mathscr{C}$. The algorithm for the construction of the set $\mathscr{C}$ is based on the topology of the power system and is presented below:

1. All the fast variables are included in $\mathscr{C}$.

2. Fast machines are detected. These are the machines which contain at least one fast variable.

3. All the variables associated to the fast machines are included in $\mathscr{C}$. 
4. Fast nodes are detected. These are the connection nodes of the fast machines and the nodes which have at least one variable in $\mathscr{C}$.

5. Propagation of the fast nodes is performed. All the nodes which are located within a given electrical distance to the fast nodes are marked as fast.

6. All the variables associated to the fast nodes are included in $\mathscr{C}$.

The presented partitioning strategy does not require much computational effort, since most of the coupling related computations can be performed once, before the time domain simulation.

\section{A chain test problem}

To illustrate the efficiency of the presented multirate strategy we use a power system composed of a chain of 100 small subsystems connected by very long lines. Each subsystem comprises a generator and the corresponding controllers modeled by 30 equations, a stepup transformer and an impedant load. A schematic illustration of the chain is presented in Figure 1. The resulting system contains 4970 variables, 3089 of which are algebraic.

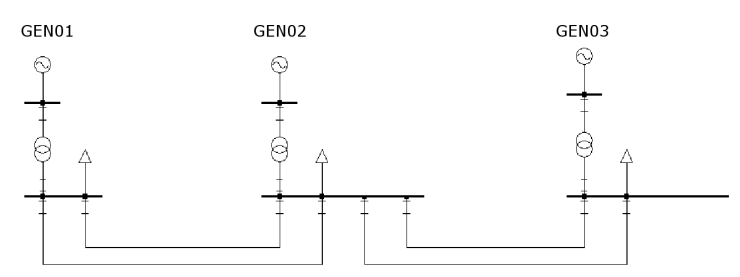

Fig. 1. Chain of 100 subsystems.

A short-circuit of $100 \mathrm{~ms}$ is performed at the first high voltage busbar. During the very first second, this event strongly affects the beginning of the chain while the rest of the system remains more or less constant. The impact of the short-circuit propagates to the neighboring subsystems while being progressively damped.

As the basic time integration method we use the mixed Adams-BDF method presented in [1]. The Adams method is applied to the differential state variables and provides a reliable detections of unstable situations. The BDF method is used for the algebraic state variables, since it less sensitive to the variations in the algebraic equations than the Adams method.

Table 1 shows the number of function evaluations and the weighted $L^{2}$ - and infinity-norm errors for the single-rate and multirate methods. From these results it is seen that a substantial improvement in number of function evaluations is obtained. For the single-rate method, the number of function evaluations is four times larger. Moreover, the error behavior of the multirate scheme is very good.

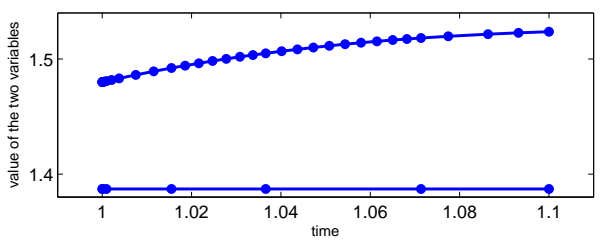

Fig. 2. Solution for two components.

Figure 2 shows the time points in which the solution for two variables, one fast and one slow, were computed. It is seen that the time steps used for the fast variable are much smaller than the ones used for the slow variable. In this simulation 70 fast variables were observed.

Table 1. Errors and number of function evaluations.

\begin{tabular}{|l|c|c|}
\hline & single-rate & multirate \\
\hline$\|$ error $\|_{\infty}$ & $7.64 \cdot 10^{-2}$ & $5.28 \cdot 10^{-2}$ \\
$\|$ | error $\|_{L^{2}}$ & $4.22 \cdot 10^{-5}$ & $4.22 \cdot 10^{-5}$ \\
nr func evals & 184326 & 47102 \\
\hline
\end{tabular}

\section{Conclusions}

In this paper we presented a multirate time stepping strategy for systems of differential and algebraic equations resulting from modeling of power systems. The algorithm for dynamic partitioning of the components into slow and fast was described. The chain test problem confirmed that the efficiency of time integration methods can be significantly improved by using large time steps for inactive components, without sacrificing accuracy.

Acknowledgement. This work was performed in the context of the PEGASE project funded by European Community's 7th Framework Programme (http://www.fp7-pegase.eu/).

\section{References}

1. J.Y. Astic, A. Bihain, and M. Jerosolimski. The mixed Adams-BDF variable step size algorithm to simulate transient and long term phenomena in power systems. IEEE Trans. Power Systems, 9:929-935, 1994.

2. J. Chen, and M.L. Crow. A variable partitioning strategy for the multirate method in power systems. IEEE Trans. Power Systems, 23:259-266, 2008.

3. V. Savcenco, W. Hundsdorfer, and J.G. Verwer. A multirate time stepping strategy for stiff ODEs. BIT, 47:137$155,2007$.

4. M. Stubbe, A. Bihain, J. Deuse, and J.C. Baader. Simulation of the dynamic behaviour of electrical power systems in the short and long terms. CIGRE, 38-03, 1998.

5. A. Verhoeven, B. Tasic, T. Beelen, E.J.W. ter Maten, and R.M.M. Mattheij. Automatic partitioning for multirate methods. In G. Ciuprina, D. Ioan (Eds), Scientific Computing in Electrical Engineering. Springer, Berlin Heidelberg New York, 229-236, 2007. 